\title{
HYDROGEN ADSORPTION AT DISLOCATIONS AND CRACKS IN Fe
}

\author{
J. P. HIRTH \\ Metallurgical Engineering Department. Ohio State University. \\ Columbus. OH 43210 . U.S.A. \\ and \\ B. CARNAHAN \\ Chemical Engineering Department. University of Michigan. \\ Ann Arbor, MI 48109. U.S.A.
}

(Received 20 Octoher 1977: in revised form 3 May 1978)

\begin{abstract}
Solute adsorption to dislocations and cracks is considered in both the Boltzmann and FermiDirac models. Explicit sums are developed for the integral amount of solute adsorbed in the defect fields. Examples of the use of the method are presented for the case of hydrogen in iron and compared with earlier results. The similarity of the condensed atmosphere of hydrogen to hydride precipitation is noted and its relevancy to hydrogen embrittlement models is discussed.
\end{abstract}

\begin{abstract}
Rúsumé-On étudie l'adsorption de soluté par les dislocations et les fissures, en utilisant les modèles de Boltzmann et de Fermi-Dirac. On obtient des sommes explicites pour la quantité intégrale de soluté adsorbée dans le champ des défauts. On présente des exemples d'application de la méthode dans le cas de l'hydrogène dans le fer, et on les compare avec des résultats antérieurs. On remarque une similitude entre l'atmosphère concensée d'hydrogène et la précipitation d'hydrure, et on discute de son incidence sur les modèles de la fragilisation par l'hydrogène.

Zusammenfassung - Im Boltzmann- und Fermi-Dirac-Modell wurde die Adsorption gelöster Atome an Versetzungen und Rissen betrachtet. Es werden explizite Summen für die integrale Menge adsorbierter Atome im Bereich der Defekte entwickelt. Beispiele für die Anwendung dieser Methode werden für den Fall des Wasserstoffs in Eisen angegeben und mit früheren Ergebnissen verglichen. Die Ähnlichkeit der kondensierten Atmosphäre von Wasserst off mit Hydridausscheidung wird aufgezeigt und deren Wichtigkeit für die Wasserstoffversprödungsmodelle wird diskutiert.
\end{abstract}

\section{INTRODUCTION}

In their original treatment of the adsorption of solute to the stress fields of dislocations. Cottrell and Bilby [1] used the Boltzmann approximation

$$
C=C_{0} \exp (W / k T)
$$

where $C$ and $C_{0}$ are the atom fractions of solute in equilibrium, respectively, in the defect field and remote from the defect, while $W$ is the interaction energy between solute and defect. Beshers [2] first noted the inadequacy of equation (1) for the case of dislocation cores, where the large ratio of $W / T$ made necessary the use of the Fermi-Dirac form

$$
\frac{C}{1-C}=\frac{C_{0}}{1-C_{0}} \exp \frac{W}{k T} \text {. }
$$

More recently the need for the use of equation (2) for other solute-dislocation problems has been widely recognized [3-5]. A particular case is that of interstitially dissolved hydrogen in boc metals where $W$ is relatively large and where permeability and diffusivity studies [6-8] indicate sufficient mobility for appreciable solute atmosphere formation at room temperature and below. Knowledge of the amount of hydrogen adsorbed at dislocations is important in assessing permeability and diffusivity of hydrogen. especially as influenced by cold work.

The integral amounts of hydrogen adsorbed to dislocations in iron at room temperature have been estimated by Bockris et al. [9] on the basis of an integral of equation (1). However, as described in Appendix 1 (see also Ref. [10]), they omitted several important terms in their series expansion, leading to an overestimate of the integral amount adsorbed by a factor of $10^{2}$. As shown in the next section, their method can be replaced by a somewhat more rapidly converging series solution to the integral using a method which is an expansion of that giving equation (14-50) in Ref. [4]. The latter solution, though. is based on equation (1) which is still likely to lead to an overestimate of the integral amounts adsorbed to dislocations.

Accordingly, in this treatment we derive the expression for the integral amount of solute adsorbed in the field of a dislocation in the Fermi-Dirac model of equation (2). In addition, we extend the earlier treatments to include the adsorption field at a planar mode I crack tip, of interest in hydrogen embrittlement problems. While the derivations and discussion 
focus on the particular case of hydrogen in iron, the results are, of course. general and apply to any solutesolvent metal case. The case of a Fermi-Dirac atmosphere of interstitial atoms in the field of a dislocation lying along a [100] direction ( $\mathrm{N}$ in $\mathrm{Fe}$ ) has previously been solved numerically by $\mathrm{Li}$ and Chou [5]. However, they do not present explicit sum solutions. Since erroneous sums have been derived for the analogous Boltzmann atmosphere case, we present the explicit sum solutions for both types of atmosphere for a dislocation and extend previous results by giving analogous explicit sum results for the mode I crack. Comparisons of the results for the two cases are useful also in delineating solute-strain field interactions which can be teated in terms of the simpler Boltzmann approximation.

\section{SOLUTE ADSORPTION FIELDS}

\subsection{Integral atmospheres}

For the edge dislocation of Fig. 1, the interaction energy between solute atoms and the dislocation in the isotropic elastic approximation is

$$
\boldsymbol{W}=A \sin \theta / r
$$

with $r, \theta$ cylindrical coordinates fixed on the dislocation and $A$ is a parameter, containing position-independent material constants, which is discussed in a following section. The integral number of solute atoms adsorbed in the elastic field of the dislocation per unit length is

$$
\frac{N}{L}=\rho \int_{0}^{2 \pi} \int_{r_{0}}^{R}\left(C-C_{0}\right) r \mathrm{~d} r \mathrm{~d} \theta,
$$

with $r_{0}$ and $R$, respectively the inner and outer cutoff radii and $\rho$ the number of solute atomic sites per unit volume. With the Boltzmann approximation of equation (1) and with equation (3), this expression becomes

$$
\begin{aligned}
\frac{N}{L}=\rho C_{0} \int_{0}^{2 \pi} & \int_{r_{0}}^{R} \\
& \times\left[\exp \left(\frac{A \sin \theta}{r k T}\right)-1\right] r \mathrm{~d} r \mathrm{~d} \theta .
\end{aligned}
$$

The integrand of equation (5) is expanded in a Taylor series expansion. Odd-powered terms in the integral over $\theta$ are set equal to zero because of the symmetry of the $\sin \theta$ functions, and even-powered terms are evaluated by item 3.621 in Ref. [11]. The integrals

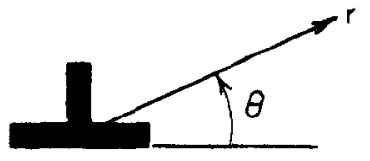

Fig. 1.

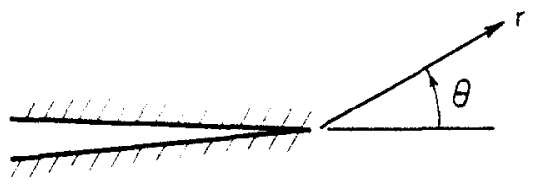

Fig. 2.

over $r$ are then straightforward with the result

$$
\begin{aligned}
\frac{N}{L}=\pi \rho C_{0} r_{0}^{2} \alpha\left\{\frac{1}{2} \ln \beta+\sum_{n=1}^{x}\right. & \\
& \left.\times \frac{1}{n\left[2^{n+1}(n+1) !\right]^{2}}\left[x^{n}\left(1-\beta^{-2 n}\right)\right]\right\} .
\end{aligned}
$$

with the non-dimensional reduced parameters $\alpha=A^{2} / r_{0}^{2} k^{2} T^{2}$ and $\beta=R / r_{0}$. Bockris et al. [9] presented a different sum for $N / L$ in this case. However. it is shown in Appendix 1 that they neglected some important contributions: when the latter are included. the two sums give identical results.

For the Fermi-Dirac distribution of equation (2), considerable manipulation is required to obtain the expression for $C-C_{0}$ to be used in equation (4). The derivation is presented in Appendix 2. leading to the result. equation (2.7). Substituting equation (2.7) into equation (4), we follow the same procedure as above. dropping odd-powered terms and so forth, with the result

$$
\begin{aligned}
& \frac{N}{L}=\pi \rho \gamma r_{0}^{2} \alpha\left[\frac{\left(1-2 C_{0}\right)\left(1-C_{0}\right)^{2}}{2} \ln \beta+\sum_{m=1}^{x}\right. \\
& \left.\times\left\{\sum_{n=1}^{x} \frac{(-1)^{m-1} \gamma^{m-1} m^{2 n+2}}{n\left[2^{n+1}(n+1) !\right]^{2}}\left[\alpha^{n}\left(1-\beta^{-2 n}\right)\right]\right\}\right],
\end{aligned}
$$

with $\gamma=C_{0} /\left(1-C_{0}\right)$.

For the crack of Fig. 2, the interaction energy between solute atoms and the elastic field of the crack is

$$
W=(B \cos \theta / 2) /{ }^{\prime} \bar{r},
$$

with $B$ a factor discussed in a later section. In the Boltzmann approximation of equation (1), equation (8) gives an expression analogous to equation (5) but with a different argument of the exponential function. The further procedure is as above. except that for this case both odd and even powers of the expansion contribute. The result is

$$
\begin{aligned}
\frac{N}{L}= & 2 \pi \rho C_{0} r_{0}^{2} \eta^{2}\left\{\frac{(\beta-1)}{4 \eta}+\frac{1}{64} \ln \beta\right. \\
& +\sum_{n=1}^{x} \frac{1}{n\left[2^{n+2}(n+2) !\right]^{2}}\left[\eta^{n}\left(1-\beta^{-n}\right)\right] \\
& +\frac{4}{\pi} \sum_{n=0}^{x} \frac{1}{(2 n-3)} \\
& \left.\times\left[\frac{2^{n} n !}{(2 n+1) !}\right]^{2}\left[\eta^{n-3 / 2}\left(1-\beta^{-n+3 / 2}\right)\right]\right\} .
\end{aligned}
$$


Here $\beta=R / r_{0}$ is retained as the ratio of cutoff radii and $\eta=B^{2} / r_{0} k T$ is the other non-dimensional parameter.

In the Fermi-Dirac case. equation (1.7) is used together with equation (8) in equation (4) with the result

$$
\begin{aligned}
L^{N}= & 2 \pi \rho \gamma r_{0}^{2} \eta^{2} \llbracket\left(\frac{\beta-1}{4 \eta}+\frac{1}{64} \ln \beta\right) \\
& \times\left(1-2 C_{0}\right)\left(1-C_{0}\right)^{2}+\sum_{m=1}^{x} \\
& \times\left\{\sum_{n=1}^{x} \frac{\gamma^{m-1} m^{2 n+4}(-1)^{m-1}}{n\left[2^{n+2}(n+2) !\right]^{2}}\left[\eta^{n}\left(1-\beta^{-n}\right)\right]\right\} \\
& +\frac{4}{\pi} \sum_{m=1}^{x}\left\{\sum_{n=0}^{x} \frac{\eta^{m-1} m^{2 n+1}(-1)^{m-1}}{(2 n-3)}\right. \\
& \left.\left.\times\left[\frac{2^{n} n !}{(2 n+1) !}\right]^{2}\left[\eta^{n-3 ! 2}\left(1-\beta^{-n+3 ! 2}\right)\right]\right\}\right] .
\end{aligned}
$$

\subsection{The interaction energy}

When the strain field of the solute atom has spherical symmetry. the interaction with the other defects is purely with the hydrostatic stress component, $\sigma_{i i} / 3$. In this case the interaction energy for the dislocation is given by $[1-4]$

$$
W=\left[\frac{\mu b_{e}(1+v) r}{3 \pi(1-v)}\right] \frac{\sin \theta}{r},
$$

with the factor in brackets defining $A$ in equation (3). Here $\mu$ is the shear modulus, $b_{e}$ is the edge component of the Burgers vector. $v$ is Poisson's ratio and $v$ is the internal increment of expansion of the solute atom. The analogous form for the mode I crack, with the hydrostatic component of the stress tensor given by Rice [12], for example. is

$$
W=\left[\left(\frac{2}{\pi}\right)^{1: 2} \frac{(1+v)}{3} K_{J} t\right] \frac{\cos \theta / 2}{v^{r}} .
$$

Here $K_{l}$ is the stress intensity, with the form $\sigma_{x} \sqrt{\pi a}$ for a uniform remote tensile stress $\sigma_{x}$ and a halfcrack length $a$ for a contained internal crack. Stress intensity factors for other external loadings are listed. for example, by McClintock and Argon [13] and by Sih [14]. The factor in brackets defines $B$ in equation (8).

These results are exact, in the isotropic elastic approximation, for substitutional solutes and for interstitials in f.c.c. metals. For interstitials in b.c.c. metals, however. there are additional interaction terms. We derive these terms for the dislocation case as an example and show that the form of equations (6) and (7) still holds while equation (3) remains a fair approximation. With other stress component interactions. the interaction energy assumes the form [15.4]

$$
W=\sigma_{i j} \epsilon_{i j} \nu
$$

where $\sigma_{i j}$ is the stress tensor of the dislocation. $V$ is the reference volume containing the solute atom and $\epsilon_{i j}$ is the local internal strain field produced by the solute atom referred to this reference volume. For either octahedral or tetrahedral site occupancy, the strain field referred to cube axes is $\epsilon_{11}>\epsilon_{22}=\epsilon_{33}$. other $\epsilon_{i j}=0$.

For a screw dislocation. the stress field is wellknown in coordinates fixed with $X_{3}^{\prime}$ parallel to the dislocation line. Transformed to cube axes (Ref. [4]. p. 471) the normal stress components, the only ones contributing to equation (13). are

$$
\begin{aligned}
& \sigma_{11}=\left(\mu b_{s} / 6 \pi r\right)(\sqrt{6} \sin \theta-\sqrt{2} \cos \theta) \\
& \sigma_{22}=\left(\mu b_{s} / 6 \pi r\right)(-\sqrt{6} \sin \theta-\sqrt{2} \cos \theta) \\
& \sigma_{33}=\left(\mu b_{s} / 6 \pi r\right)(2 \sqrt{2} \cos \theta)
\end{aligned}
$$

Substituting in equation (13). we find

$$
W=\left[\frac{\mu b_{s} V_{v} \overline{6}}{6 \pi}\left(\epsilon_{11}-\epsilon_{22}\right)\right] \frac{\sin \theta}{r}\left(1-\frac{\overline{3}}{3} \cot \theta\right) \text {. }
$$

where $b_{s}$ is the screw component of the Burgers vector. There are three types of interstitial sets with $\epsilon_{11}$ defined alternatively along each of the cube axes and with differing local energies, but they all give the same contribution when integrated over $\Delta \theta=2 \pi$, so equation (15) suffices for all three. If the factor in brackets is identified with $A$ in equation (3). the term including $\cot \theta$ then represents the correction to eqution (3) for this case. Proceeding with the development as in the previous section. we find that the integral over $\theta$ has the same form (Ref. [11]. item 3.661) as the previous case, but with an additional, $\theta$-independent factor. The result is the same as for equations (6) and (7) except that the sum over $n$ contains the additional factor $4(4 / 3)^{n+1}$.

The edge dislocation case is considerably more complicated and is presented in Appendix 3. Even for the edge case. however. the approximation of equations (6) and (7) is shown to be fairly good.

\subsection{The volume expansion}

The strength of volume expansion $v$ to be used in equation (11) and the corresponding cube displacements $\epsilon_{11} V, \epsilon_{22} V$ and $\epsilon_{33} V$ are related to the internal. local expansion on formation of the solute atom [16-18]. The external volume expansion contains an image expansion factor $3(1-v) /(1+v)$. Hence, contrary to earlier treatments. the relation with the thermodynamic quantity $\vec{V}$, the partial molar volume of the solute, is

$$
v=\bar{V}(1+v) / 3 N_{A}(1-v) .
$$

where $N_{A}$ is Avagadro's number. The assignment of $\bar{V}$ itself is a problem in interstitial systems, in particular for hydrogen in iron, as discussed in detail elsewhere [10]. The quantity $\bar{V}$ is often determined from experimental measurements of the change in solu- 
bility with pressure

$$
\vec{V}=-k T\left(\hat{C} \ln C_{0} / \partial P\right)
$$

However, because of experimental difficulties with low-temperature interstitial systems, the measurements are often made for specimens under simple tension and $P$ is taken as $-\sigma_{i i} / 3=-\sigma_{x} / 3$. In this case, however, other stress components can effect the result via terms analogous to those in equation (13). Hence the $\bar{V}$ itself may only be an apparent value: estimates of the effect [10] suggest that the apparent value can exceed the true value of $\bar{V}$ by as much as $60 \%$ the equivalent in the present work would be a shift in $A$ by this amount from equation (11) to equation (3.2)).

\subsection{Solute site density}

For a substitutional solute, $\rho$ is simply the number of atomic sites per unit volume and saturation corresponds to a region of pure solute. For the interstitial case, $\rho$ can differ from the atomic site density both intrinsically and because of site blocking [19-20]. For f.c.c. crystals there are two tetrahedral sites and one octahedral site per solvent atom while for b.c.c. crystals there are six tetrahedral sites and three octahedral sites per solvent atom. Hence sufficient site occupancy to correspond to compounds $\mathrm{MX}_{3}$ and $\mathrm{MX}_{9}$ would be possible in principle. However, site exclusion of elastic or electronic interactions in general reduces the maximum occupancy. For the case of iron-hydrogen, of particular interest here, there are no thermodynamically stable hydrides. However, a recent survey by Speiser [21] shows that other transition metals have hydrides with compositions $\mathrm{MH}_{2}$ (mainly rareearth metals with large atomic size) and $\mathrm{MH}$. Accordingly, we select a value of $\rho$ for the iron-hydrogen case equal to the number of iron atom sites per unit volume. equivalent to a site-saturated composition FeH. As is evident from equation (1), we assume that there is no interaction energy between hydrogen atoms. This assumption is consistent with thermodynamic data for hydrogen solubility [20], but may not apply as site saturation is approached. However. data are not available for the interaction energy. which would also be required for an accurate estimate of site exclusion as discussed above. so the interaction energy is taken as zero.

\section{PROCEDURE}

As an example of the comparison between the Boltzmann and Fermi-Dirac solute distributions, we select hydrogen in iron at $298 \mathrm{~K}$. For the material parameters in $A$ in equation (3), we take the Voigt average elastic constant values [4] $\mu=86 \mathrm{GPa}$. $\gamma=0.29 ;$ the partial volume of hydrogen in iron [9] $\bar{V}=2.66 \times 10^{-6} \mathrm{~m}^{3} / \mathrm{mol}$. and $v=\bar{V}$. giving $(A / k T)=4.416 \mathrm{~nm}$. With $r_{0}=b=0.248 \mathrm{~nm}$ this would give a value of $x=317.1$. so $x$ values in this range were selected. On the basis of the work of Gon- zales [22], the concentration $C_{0}$ at $298 \mathrm{~K}$ is $3.5 \times 10^{-8}$ in equilibrium with a hydrogen pressure $p=1.013 \times 10^{5} \mathrm{~Pa}(1 \mathrm{~atm})$. A typical value of the enhanced concentration produced by electrolytic charging is $C_{0}=10^{-3}$ at $298 \mathrm{~K}$. Hence values of $C_{0}=\gamma=5 \times 10^{-8}$ and $10^{-9}$ were examined to span the room temperature range of hydrogen concentrations encountered in practice. In order to evaluate $N / L, \rho$ was taken as $1.409 \times 10^{5} \mathrm{~mol} / \mathrm{m}^{3}$.

For the parameter $B$ in equation (8), the above values were selected together with $K_{I}=0.3494$ $\mathrm{MPa}, \overrightarrow{\mathrm{m}}$, corresponding to a value of the local stress equal to the theoretical perfect crystal strength of $E / 10$, with $E$ the Voigt average value of Young's modulus, at $r=b$. These values result in $B^{2} / k T=$ $16.53 \mathrm{~nm}$, which for $r_{0}=0.248 \mathrm{~nm}$ gives $\eta=66.65$, so values in this range were selected for computation. These values correspond to the purely brittle crack case for iron. Also, for a plastically-relaxed crack in an iron-based alloy, the recent model of Thomson [23] indicates that the crack tip is screened from relaxation over spacings of the order of the values selected here for $R$. Thus, the present results also apply to the case considered by Thomson [23].

With the above values, the several sums were evaluated by numerical methods.

\section{RESULTS AND DISCUSSION}

Results for the dislocation case are presented in Table 1. For this case. the results for the Fermi-Dirac solution show divergence at values of $x$ somewhat below the value $x=317$ corresponding to the case $r_{0}=0.248 \mathrm{~nm}$ previously considered for the Boltz mann solution $[9,10]$, with divergence at a relatively smaller $x$ value for the larger value of $C_{0}$. This indicates condensation to a nearly site saturated solution in the vicinity of $r_{0}$. For these cases. therefore, the smaller values of $x$ for which a convergent solution obtains, and a correspondingly larger value of $r_{0}$. must be selected to obtain specific results for $N / L$ the hydrogen atmosphere in the remote strain field. The appropriate $r_{0}$ values for the $x$ values in Table 1 (and for the $\eta$ values in Table 2) are listed in Table 3. The results show that the first-term approximation in Ref. [4] gives a poor estimate of $N / L$. However, use of the simpler Boltzmann series solution is seen to give a fairly accurate estimate of the Fermi-Dirac solution in all cases. the maximum deviation being four percent.

The results for the crack case are presented in Table 2. In this case convergence is obtained for $\eta$ values larger than that. 66.6, corresponding to $r_{0}=0.248$. As for the dislocation case, the simpler Boltzmann result gives a fairly accurate estimate of the Fermi-Dirac result, while the first term approximation is very poor.

The contribution of the remote stress field atmosphere to the apparent enhancement in hydrogen solu- 


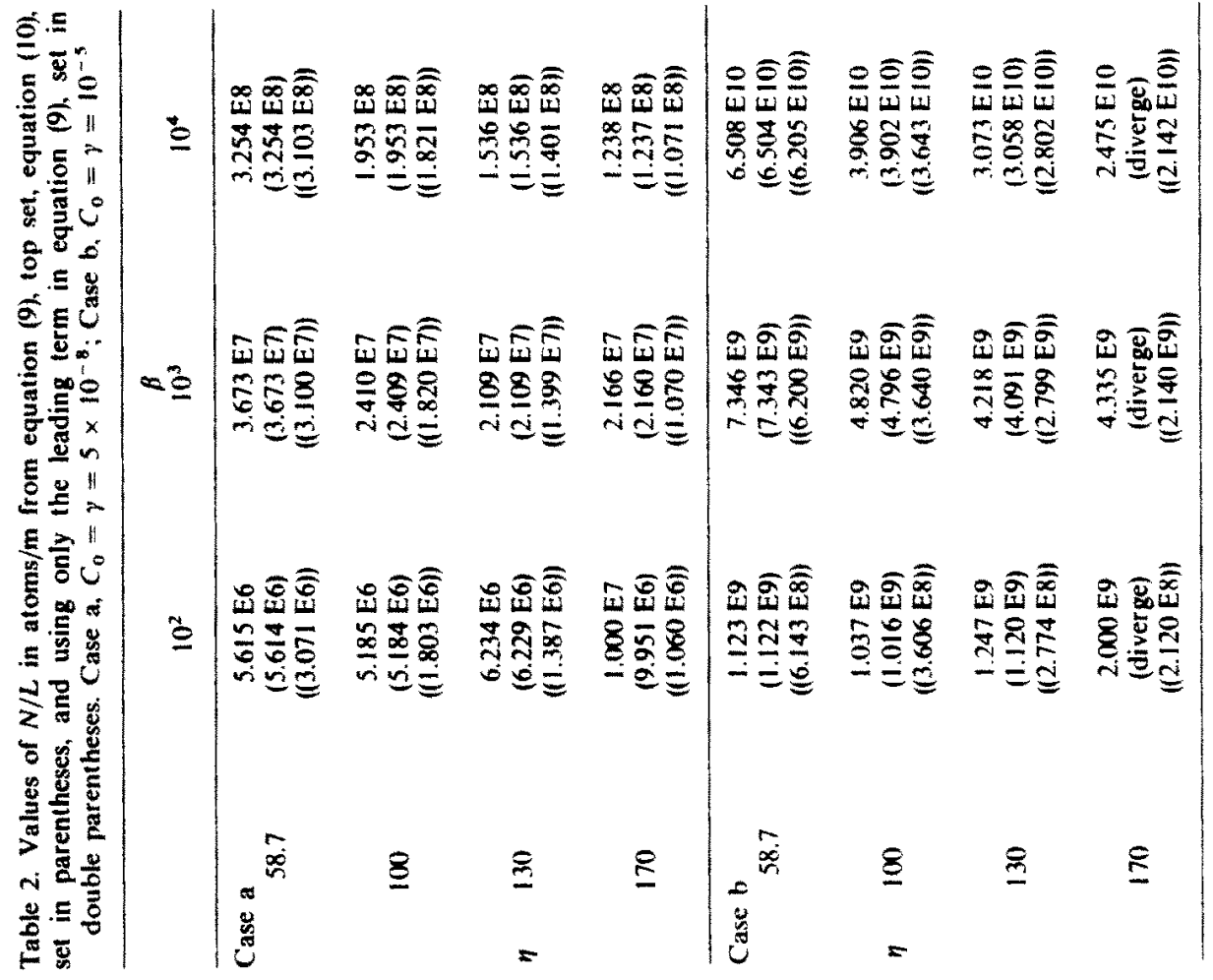

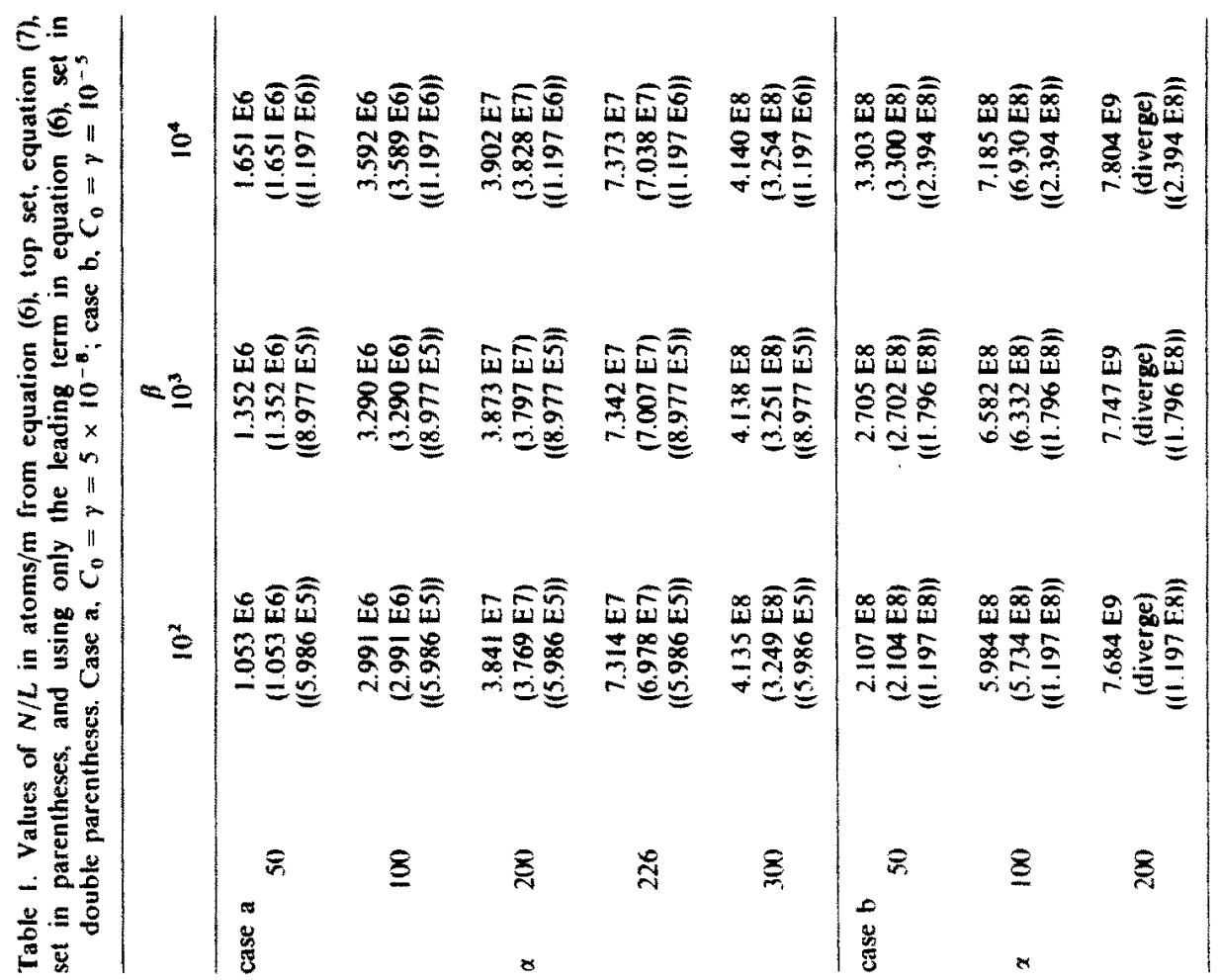


Table 3. Values of $r_{0}, A / k T$ and $B^{2} / k T$ in $\mathrm{nm}$. corresponding to the $x$ and $\eta$ values listed in Tables 1 and 2

\begin{tabular}{cccccc}
\hline$x$ & 50 & 100 & 200 & 226 & 300 \\
\hline$r_{0}$ & 0.625 & 0.442 & 0.312 & 0.293 & 0.255 \\
$A / k T$ & 4.416 & 4.416 & 4.416 & 4.416 & 4.416 \\
\hline & & & & & \\
\hline$\eta$ & 58.7 & 100 & 130 & 170 \\
\hline $\begin{array}{c}r_{0} \\
B^{2} / k T\end{array}$ & 0.282 & 0.165 & 0.127 & 0.097 \\
\hline
\end{tabular}

bility or permeability can be calculated by comparing $N / L$ with $N_{0} / L$, the amount of hydrogen that would be present in an unstrained cylindrical section of inner and outer radii $r_{0}$ and $R$, respectively. The contribution to the mean hydrogen concentration would be proportional to $N / L$ for a dislocated crystal with dislocations spaced by a distance $R$ so the enhancement of concentration would be a factor $N / N_{0}$. For a crack, $N / N_{0}$ represents a local hydrogen enhancement factor. Table 4 presents such calculations. For dislocations the enhancement is modest in comparison to earlier estimates [9] because of the smaller $x$ values required for a convergent solution. The enhancement is important only for smaller $\beta$ values, corresponding to large dislocation densities or highly work hardened material, or to material near a crack tip. For example. the dislocation case with an enhancement factor of 6.105 corresponds to a disiocation density of $1.16 \times 10^{11} \mathrm{~cm}^{-2}$.

Within a distance $r_{0}$ of the defect, a "core" region excluded in the calculation of $N / L$, one must perform atomic calculations for the hydrogen concentration. Use of the Fermi-Dirac model is crucial in this region where the atmosphere is nearly saturated, a point not included in the work of Bockris et al. who obtained a result for a single-site core atmosphere which was an overestimate because of the use of a Boltzmann approximation for the calculation [10]. For core regions of interest here, with $r_{0}$ ranging up to $0.442 \mathrm{~nm}$. the interstitial sites within the cores are those listed in Table 5. For the dislocation case, for example. Table 5 shows that up to 96 sites per repeat distance may require atomic calculation. No such estimates are attempted here since the requisite atomic potential is unavailable. but the table does give the upper limit of the hydrogen concentration enhancement in the core. For the case where $N / N_{0}$ is 6.105 in Table 4 for example. the limiting core factor is associated with 42 sites per repeat distance, corresponding to $N_{\text {vured }} / N_{0}=1.48 \times 10^{4}$.

The site saturation results for the crack are pertinent to the mechanism for hydrogen embrittlement suggested by Birnbaum et al. [24] and verified by them $[25,26]$ for the case of hydrogen embrittlement of niobium. In this model, the hydrostatic tension field at the crack tip can modify the free energy of formation of a hydride so that. while unstable in the absence of stress, it becomes stable at the crack tip. Fracture then proceeds by a cyclic process of cracking of the hydride, crack arrest and reformation of the hydride. On the basis of the present results, site saturation to some level corresponds to hydride formation. As stated before, atomic calculations with site exclusion effects associated with hydrogen-hydrogen interactions would be required for an exact solution to the problem. In a macroscopic model. such interactions would contribute to the bulk and surface free energies of the hydride. A rough estimate of the size of such a region can be made on the basis of the present results. Listed in Table 6 are values of $r$, calculated from equations (3) and (8) along the value of $\theta$ where maximum elastic interaction occurs, at which the Fermi-Dirac calculation predicts a local hydrogen/iron ratio of $1,1 / 4,1 / 16$ and 1/64. Known hydride compositions for transition metals are encompassed in this range. Even for the stringent case of a ratio of 1 , the condensed region is seen to extend over a distance of $4-5 a_{0}$ and with reference to Table 5 , to incorporate a large number of interstitial

Table 4. Values of $N_{0} / L$ and $N / N_{0}$ for several cases

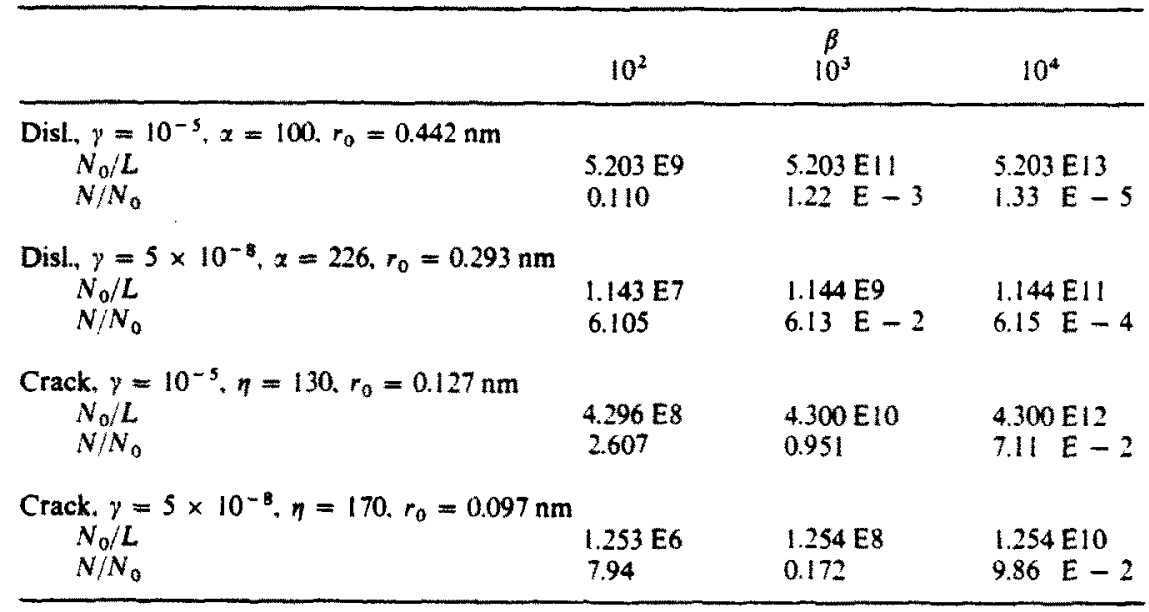


Table 5. Site table for interstitial site distribution about: an atom in a perfect crystal: per repeat distance $a_{0}$ along the line of a $[100](001)$ crack; and per repeat distance, $\overline{3} a_{0} / 2$ along a [111] screw dislocation. Site vectors in units of one-half lattice parameter. $a_{0}$

\begin{tabular}{|c|c|c|c|c|c|c|}
\hline $\begin{array}{l}\text { Type } \\
\text { of } \\
\text { site } \\
\text { vector }\end{array}$ & $\begin{array}{r}\text { Pe } \\
\text { No. of } \\
\text { sites }\end{array}$ & $\begin{array}{l}\text { Stal } \\
\text { Site } \\
\text { spacings } \\
(n m)\end{array}$ & $\begin{array}{c}\quad[100] \\
\text { Sites } \\
\text { per repeat } \\
\text { distance }\end{array}$ & $\begin{array}{l}\text { ack } \\
\qquad \begin{array}{c}r \\
(\mathrm{~nm})\end{array}\end{array}$ & $\begin{array}{l}\text { [111] S } \\
\text { Sites per } \\
\text { repeat } \\
\text { distance }\end{array}$ & $\begin{array}{l}\text { ocation } \\
\qquad \begin{array}{c}r \\
(\mathrm{~nm})\end{array}\end{array}$ \\
\hline$[100]$ & 6 & 0.143 & $\frac{2}{3}$ & $\begin{array}{l}0 \\
0.143\end{array}$ & 6 & 0.117 \\
\hline$[110]$ & 12 & 0.202 & $\begin{array}{l}4 \\
4\end{array}$ & $\begin{array}{l}0.143 \\
0.202\end{array}$ & $\begin{array}{l}6 \\
6\end{array}$ & $\begin{array}{l}0.185 \\
0.234\end{array}$ \\
\hline$[210]$ & 24 & 0.320 & $\begin{array}{r}6 \\
16\end{array}$ & $\begin{array}{l}0.286 \\
0.320\end{array}$ & $\begin{array}{l}12 \\
12\end{array}$ & $\begin{array}{l}0.202 \\
0.309\end{array}$ \\
\hline$[121]$ & 24 & 0.350 & 12 & 0.320 & $\begin{array}{r}6 \\
12\end{array}$ & $\begin{array}{l}0.248 \\
0.309\end{array}$ \\
\hline$[300]$ & 6 & 0.429 & 3 & 0.429 & 6 & 0.248 \\
\hline$[212]$ & 24 & 0.429 & 6 & 0.404 & $\begin{array}{r}6 \\
12\end{array}$ & $\begin{array}{l}0.305 \\
0.421\end{array}$ \\
\hline$[310]$ & 24 & $\cdot 0.452$ & $\begin{array}{l}6 \\
6\end{array}$ & $\begin{array}{l}0.429 \\
0.452\end{array}$ & 12 & 0.421 \\
\hline$[320]$ & 24 & 0.516 & 6 & 0.516 & 12 & 0.509 \\
\hline$[3 \mid 2]$ & 48 & 0.535 & 12 & 0.516 & $\begin{array}{l}12 \\
12\end{array}$ & $\begin{array}{l}0.509 \\
0.584\end{array}$ \\
\hline$[410]$ & 24 & 0.590 & 6 & 0.590 & 12 & 0.535 \\
\hline [322] & 24 & 0.590 & 6 & 0.572 & $\begin{array}{l}12 \\
12\end{array}$ & $\begin{array}{l}0.535 \\
0.584\end{array}$ \\
\hline [330] & 12 & 0.607 & 3 & 0.607 & 6 & 0.555 \\
\hline [141] & 24 & 0.607 & 12 & 0.590 & $\begin{array}{r}12 \\
6\end{array}$ & $\begin{array}{l}0.509 \\
0.584\end{array}$ \\
\hline [241] & 48 & 0.655 & 12 & 0.640 & $\begin{array}{l}12 \\
12\end{array}$ & $\begin{array}{l}0.607 \\
0.650\end{array}$ \\
\hline
\end{tabular}

sites. Thus. the present results indicate that on a near atomic scale in iron the model of Birnbaum et al. [24] is possible as the specific cracking portion in the embrittlement mechanism proposed by Thomson [23].

\section{SUMMARY}

In summary, series solutions are presented for both Boltzmann and Fermi-Dirac atmospheres of solute atoms in the strain fields of dislocations and cracks. The solutions applied to the case of hydrogen in iron yield results differing markedly from previous results. Beyond a critical inner cutoff radius for convergence of the series solutions. the Boltzmann result gives a fair approximation of the Fermi-Dirac result. Within the core cutoff radius, use of the Fermi -Dirac model is necessary. extending over many interstitial sites: as many as 96 per repeat distance along a dislocation in the cases considered. Estimates of the extent, of the condensed Fermi-Dirac atmosphere of hydrogen in iron indicate that the embrittlement models of both Birnbaum et al. [24] and Thomson [23] apply to this case.

Table 6. Values of $r$ in $\mathrm{nm}$ where $C$ equals specific values for the maximum interaction cases where $\theta=\pi / 2$. equation (3). and $\theta=0$. equation (8)

\begin{tabular}{lcccc}
\hline & 1 & $1 / 4$ & $1 / 16$ & $1 / 64$ \\
\hline $\begin{array}{l}C_{0}=\gamma=5 \times 10^{-8} \\
\text { equation (3) }\end{array}$ & 0.262 & 0.286 & 0.315 & 0.349 \\
$\begin{array}{l}C_{0}=\gamma=10^{-5} \\
\text { equation (3) }\end{array}$ & 0.383 & 0.436 & 0.505 & 0.601 \\
$\begin{array}{l}C_{0}=5 \times 10^{-8} \\
\text { equation (8) }\end{array}$ & 0.944 & 1.03 & 1.12 & 1.24 \\
$\begin{array}{l}C_{0}=10^{-5} \\
\text { equation (8) }\end{array}$ & 1.35 & 1.53 & 1.75 & 2.05 \\
\hline
\end{tabular}


Acknowledgement-This research was supported in part by the Defense Advanced Research Projects Agency under contract DAHC 15-71-C-0253 with the University of Michigan and in part by National Science Foundation Grant DMR-74-21243 with the Ohio State University.

\section{REFERENCES}

1. A. H. Cottrell and B. A. Bilby, Proc. Phys. Soc., Lond. A62. 49 (1949).

2. D. N. Beshers, Acta metall. 6, 521 (1958).

3. F. R. N. Nabarro, Theory of Crystal Dislocations, p. 403. Oxford University Press (1967).

4. J. P. Hirth and J. Lothe, Theory of Dislocations, p. 466. McGraw-Hill. New York (1968).

5. J. C. M. Li and Y. T. Chou, Trans. Met. Soc. A.I.M.E. 245. 1606 (1969).

6. W. Beck, J. O'M. Bockris, J. McBreen and L. Nanis, Proc. R. Soc. A290, 220 (1966).

7. T. K. G. Namboodhiri and L. Nanis. Acta metall. 21, $663(1973)$

8. A. J. Kumnick and H. H. Johnson, Acta metall. 25, 891 (1977).

9. J. O'M. Bockris, W. Beck, M. A. Genshaw, P. K. Subramanyan and F. S. Williams, Acta metall. 19, 1209 (1971).

10. J. P. Hirth, In: Stress Corrosion Cracking and Hydrogen Embrittlement of Iron-Based Alloys, (edited by $\mathbf{R}$. W. Staehle et al.) Natn. Ass. Corrosion Engng. Houston. Texas (1977).

i. I. S. Gradshteyn and I. M. Ryzhik, Table of Integrals, Series and Products. Academic Press. New York (1965).

12. J. R. Rice, In: Fracture. Vol: II, p. 191. Academic press, New York (1968).

13. F. A. McClintock and A. S. Argon, Mechanical Behavior of Materials. p. 406. Addison-Wesley, New York (1966).

14. G. C. Sih, Methods of Analysis and Solutions of Crack Problems. Noordhoff. Netherlands (1973).

15. A. W. Cochardt. S. Schoeck and H. Wiedersich. Acta metall. 3, 533 (1955).

16. J. Weertman. Phil. Mag. 11. 1217 (1965)

17. J. Lothe and J. P. Hirth, J. appl. Phys. 38, 845 (1967)

18. J. Lothe and J. P. Hirth, J. appl. Phys. 38, 4916 (1967)

19. R. Speiser and J. W. Spretnak, Trans. A.S.M. 47, 493 (1955).

20. K. Alex and R. B. McLellan, Acta metall. 21, 107 (1973).

21. R. Speiser. In: Stress Corrosion Cracking and Hydrogen Embrittlement of Iron-Based Alloys, (edited by R. W. Staehle et al.) Natn. Ass. Corrosion Engng, Houston, Texas (1977).

22. O. D. Gonzalez (quoted by R. A. Oriani) In: Fundamental Aspects of Stress Corrosion Cracking, p. 32. Natn. Ass. Corrosion Engng, Houston. Texas (1969).

23. R. M. Thomson. J. Mater. Sci. 13, 128 (1978).

24. H. K. Birnbaum, M. L. Grossbeck and S. Gahr, In: Hydrogen in Metals, p. 303. Am. Soc. Met., Metals Park, Ohio (1974).

25. S. Gahr, M. L. Grossbeck and H. K. Birnbaum, Acto metall. 25. 125 (1977).

26. M. L. Grossbeck and H. K. Birnbaum, Acta metall 25. 135 (1977)

\section{APPENDIX 1}

Comparison of equation (6) with previous series solutions

Bockris et al. [9] presented a series solution to equation (5). In their solution, they correctly evaluated the indefinite integral over dr but then neglected several terms in $r_{0}$ and
$\boldsymbol{R}$ on inserting the limits. Keeping these terms, one finds that their equation (27) acquires additional terms

$C_{\mathrm{a}}=C_{\mathrm{o}}\left[-\beta^{-2} I_{0}(D)-\alpha^{1 / 2} \beta^{-2} I_{1}(D)\right.$

$$
\left.+I_{0}(D / \beta)+\alpha^{1 / 2} \beta^{-1} I_{1}(D / \beta)\right] \text {. }
$$

where $I_{0}$ and $I_{1}$ are modified Bessel functions of order zero and one, respectively and $D=A / r_{0} k T$. The integrals leading to equation (1.1) are of the form

$$
\int_{0}^{2 \pi} \exp (D \sin \theta) \mathrm{d} \theta \text {, and } \int_{0}^{2 \pi} \sin \theta \exp (D \sin \theta) \mathrm{d} \theta \text {. }
$$

The first integral is given by item 3.387 .1 and the second by item 8.431 .5 in Ref. [11].

For the case treated by Bockris et al. [9], i.e. $A / k T=17.2 r_{0}, R=10^{3} r_{0}$, their equation (27) gives the result $C=51.9478 C_{0}$. Equation (1.1) for the same parameters gives the result $C_{a}=-50.5196 C_{0}$. The correct result is thus $C=1.4282$ which is considerably smaller and gives a result for $N / L$ a factor of 121 smaller than that of Ref. [9].

A simpler approach than modifying the earlier result in this manner, however, in the sense of producing a more rapidly converging series, is to extend the series expansion on p. 464 of Ref. [4], giving the result presented in equation (6).

\section{APPENDIX 2}

Evaluation of $\left(C-C_{0}\right)$ in the Fermi-Dirac case

We define the quantities $x=W / k T$ and $y=\exp x-1$, in terms of which equation (2) reduces to

$$
\left(C-C_{0}\right) /\left(1-C_{0}\right)=y /\left(y+C_{0}^{-1}\right)
$$

Division of the right side of equation (2.1) gives

$$
\left(C-C_{0}\right) /\left(1-C_{0}\right)=\left(C_{0} y-C_{0}^{2} y^{2}+C_{0}^{3} y^{3}-\ldots\right) \text {. }
$$

Expanding equation (2.2) in terms of $x$, we find

$$
\begin{aligned}
\frac{C-C_{0}}{1-C_{0}}= & \left(-C_{0}-C_{0}^{2}-\cdots-C_{0}^{n}\right) \\
& +\exp x\left(C_{0}+2 C_{0}^{2}+\cdots+n C_{0}^{n}\right) \\
& -\exp 2 x\left(C_{0}^{2}+3 C_{0}^{3}+\cdots+n(n-1) C_{0}^{n} / 2 !\right) \\
& +\exp 3 x\left(C_{0}^{3}+\cdots\right)+\cdots .
\end{aligned}
$$

Each of the sums in $C_{0}$ can be evaluated explicitly by manipulation of the basic result, item 0.231 in Ref. [11]

$$
\sum_{n=0}^{\infty} C_{0}^{n}=1 /\left(1-C_{0}\right) \text {. }
$$

Differentiating equation (2.4) with respect to $C_{0}$ and multiplying by $C_{0}$ we have

$$
C_{0} \sum_{n=0}^{\infty} n C_{0}^{n-1}=\sum_{n=0}^{\infty} n C_{0}^{n}=C_{0} /\left(1-C_{0}\right)^{2} \text {. }
$$

Further differentiation gives the other sums in equation (2.3). which yields

$$
\left(C-C_{0}\right)=-C_{0}+\gamma e^{x}-\gamma^{2} e^{2 x}+\gamma^{3} e^{3 x}-\ldots,
$$

where $\gamma=C_{0} /\left(1-C_{0}\right)$. Finally, expanding the exponential factors in equation (2.6) and gathering terms, we find

$$
C-C_{0}=\sum_{n=1}^{\infty} \frac{x^{n}}{n !} f_{n}(\gamma) \text {. }
$$

where

$$
f_{n}(\gamma)=\sum_{m=1}^{\infty} m^{n} \gamma^{m}(-1)^{m-1} .
$$

Equation (2.8) can be re-expressed as a finite sum of $m / 2$ terms. However, no simple recursion formula could be found for the latter sum, so there is no advantage in using it. 


\section{APPENDIX 3}

Interaction energy between interstitial and edge dislocation In this case the dislocation stress field is well known $[3,4]$ in coordinates fixed on the dislocation with $X_{1}^{\prime}$ parallel to the Burgers vector $b_{k}$ and $X_{3}^{\prime}$ parallel to the sense vector $\xi$ and with $X_{1}^{\prime} / /[111]$. $X_{2}^{\prime} / /[110]$. $X_{3}^{\prime} / /[\overline{112}]$. Transformed to cube axes. the normal stresses which contribute to equation (13) are

$$
\left.\begin{array}{rl}
\sigma_{11}= & \frac{\mu b}{12 \pi(1-v) r}[(3+2 v) \sin \theta \\
& \left.+2 \sin ^{3} \theta+2 \sqrt{6} \cos \theta \cos 2 \theta\right] \\
\sigma_{22}= & \frac{\mu b}{12 \pi(1-v) r}[(3+2 v) \sin \theta \\
& \left.+2 \sin ^{3} \theta-2, \overline{6} \cos \theta \cos 2 \theta\right] \\
\sigma_{33}= & \frac{\mu b}{6 \pi(1-v) r}\left[(3+4 v) \sin \theta-2 \sin ^{3} \theta\right]
\end{array}\right\}
$$

In the subsequent integration all terms containing the factor $\cos \theta \cos 2 \theta$ integrate to zero. Hence, we drop the latter term and present a reduced interaction energy. As discussed for the screw dislocation, there are three types of interstitial site with the appropriate stress feld given by cyclic permutation of equation (3.1).

The interaction energies given by substitution into equation (13) are of the form

$$
W=\left[\frac{\mu b V g}{12 \pi(1-v)}\right] \frac{\sin \theta}{r}\left(1+h \sin ^{2} \theta\right) .
$$

Two of the sites have the same interaction energy with

$$
\begin{aligned}
& g=(3+2 v)\left(\epsilon_{11}+\epsilon_{22}\right)+2(3+4 v) \epsilon_{33} . \\
& h=\left[2\left(\epsilon_{11}+\epsilon_{22}\right)-4 \epsilon_{33}\right] / g .
\end{aligned}
$$

The other site has

$$
\begin{aligned}
& g=2(3+4 v) \epsilon_{12}+(3+2 v)\left(\epsilon_{22}+\epsilon_{33}\right) . \\
& h=\left[-4 \epsilon_{11}+2\left(\epsilon_{22}+\epsilon_{33}\right)\right] / g .
\end{aligned}
$$

Identifying the factor in brackets in equation (3.2) with $A$ in equation (3). we see that the factor containing $\sin ^{2} \theta$ is the correction factor for this case. Proceeding as in the earlier examples. we find that the result is given by equations (6) and (7) with the additional factor for the $n$th term in the sum over $n$

$$
\begin{aligned}
{[1+} & H(2 h-1+\delta) 2 n h \\
& +H(2 n-4+\delta)(2 n-2)(2 n-1) h^{2} 2 ! \\
& +\ldots+H(2 n-1-3 m+\delta)(2 n-2 m+2) ! h^{m} / 2 ! \\
& (2 n-m) !] .
\end{aligned}
$$

Here $0<\delta<1$ and $H$ is the heaviside operator: e.g. $H(2 n-1+\delta)=0$ for $2 n-1<0 . H(2 n-1+\delta)=1$ for $2 n-1>0$

Evidently, equation (3.2) reduces to equation (11) and $h=0$ when $\epsilon_{11}=\epsilon_{22}=\epsilon_{33}=e / 3$ with $e=v / l$ equal to the dilatational component of strain. Even for $\epsilon_{11} \neq \epsilon_{22}=\epsilon_{33}$, however. the corrections to equations $(6)$ and (7) are minor except for very nearly saturated atmos. pheres where the series converge very slowly. Since this case has an obvious (saturated) answer anyway. the approximations of equations (6) and (7) are fairly good. 